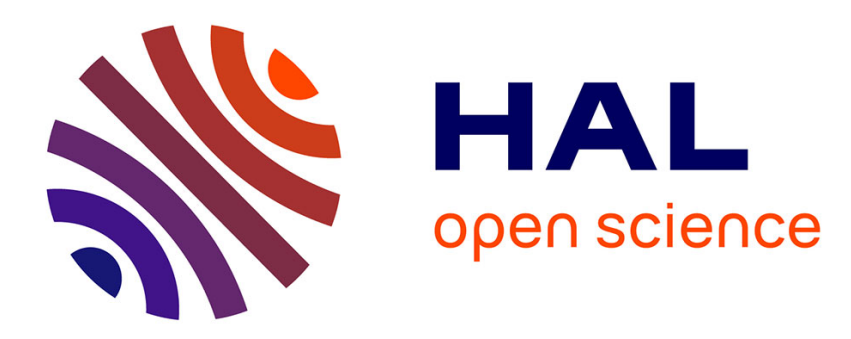

\title{
Une charge active pour photopiles solaires
}

\author{
J.R. Leguerre, G. Lavertu
}

\section{To cite this version:}

J.R. Leguerre, G. Lavertu. Une charge active pour photopiles solaires. Revue de Physique Appliquée, 1982, 17 (12), pp.807-812. 10.1051/rphysap:019820017012080700 . jpa-00245061

\section{HAL Id: jpa-00245061 https://hal.science/jpa-00245061}

Submitted on 1 Jan 1982

HAL is a multi-disciplinary open access archive for the deposit and dissemination of scientific research documents, whether they are published or not. The documents may come from teaching and research institutions in France or abroad, or from public or private research centers.
L'archive ouverte pluridisciplinaire HAL, est destinée au dépôt et à la diffusion de documents scientifiques de niveau recherche, publiés ou non, émanant des établissements d'enseignement et de recherche français ou étrangers, des laboratoires publics ou privés. 
Classification

Physics Abstracts

07.50

\title{
Une charge active pour photopiles solaires $(*)$
}

\author{
J. R. Leguerre (**) et G. Lavertu \\ Faculté des Sciences, Département de physique, Av. Ibn Batota, Rabat, Maroc
}

(Reçu le 25 juin 1982, accepté le 23 septembre 1982)

\begin{abstract}
Résumé. - Le point de puissance maximale $P_{M}$ d'une cellule solaire varie suivant le niveau d'éclairement et le type de cellule utilisée.

Les auteurs présentent la réalisation d'un appareil dont la fonction principale est la détermination de cette puissance $P_{\mathrm{M}}$ ainsi que ses coordonnées courant et tension à chaque instant de la journée.

La particularité de leur étude est la mise au point d'un dispositif de fonctionnement sûr, réalisable avec des composants ordinaires de faible coût, notamment au niveau du circuit de multiplication où les composants intégrés assurant la même fonction sont d'un prix relativement plus élevé.
\end{abstract}

\begin{abstract}
Power maximum point $P_{M}$ of a solar cell varies with sunlight level and type of device.
We present an apparatus whose main function is the determination of this power $P_{M}$ at all times of the day.

One peculiarity of the study is to have an apparatus with safe operation using cheap electronic components in the multiplication circuit where IC can serve same function but are more expensive.
\end{abstract}

1. Introduction. - Une cellule solaire est un générateur d'énergie électrique dont la caractéristique courant-tension $I(V)$ est représentée par la courbe en trait plein de la figure 1 pour un éclairement donné $\varepsilon$. $I_{\text {cc }}$ et $V_{\text {oc }}$ sont le courant de court-circuit et la tension en circuit ouvert respectivement. Le point $P_{\mathrm{M}}$ de coordonnées $V_{\mathrm{M}}, I_{\mathrm{M}}$ correspond à la puissance maximale. Pour un autre niveau d'éclairement $\varepsilon^{\prime}>\varepsilon$, la caractéristique $I(V)$ est représentée par la courbe en pointillé sur laquelle on remarque que le point de puissance maximale $P_{\mathrm{M}}$ s'est déplacé en $P_{\mathrm{M}}^{\prime}$.

Au cours d'une journée, les variations en fonction du temps du point $P_{\mathrm{M}}$ dépendent non seulement des fluctuations du rayonnement incident, mais encore des caractéristiques $I(V)$ propres à chaque dispositif étudié. Si l'on veut faire des comparaisons de rendement entre plusieurs photopiles éclairées par le même rayonnement, il est nécessaire de disposer d'appareils qui peuvent donner à chaque instant, les valeurs de $P_{\mathrm{M}}$ de chaque cellule étudiée.

L'instrumentation présentée dans cet article, répond à cette préoccupation. Il fournit en permanence les coordonnées $P_{\mathrm{M}}, V_{\mathrm{M}}, I_{\mathrm{M}}$ sous forme de tensions électriques. Il est ainsi possible par l'intermédiaire d'un enregistreur graphique de tracer les évolutions de

( $^{*}$ La nomenclature des composants apparaissant dans les figures $4,5,6,7,8$ et 9 peut être obtenue sur simple demande aux Editions de Physique.

$\left({ }^{* *}\right)$ Adresse permanente en Fraice : LAAS, 7, avenue du Colonel Roche, 31400 Toulouse.

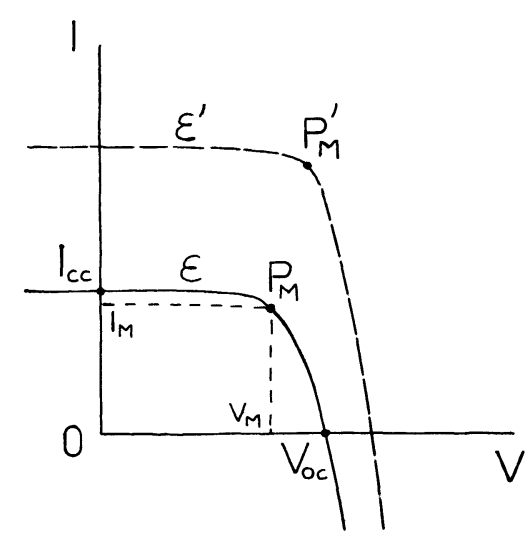

Fig. 1. - Caractéristiques courant-tension $I(V)$ d'une photopile solaire pour 2 niveaux d'éclairement $\varepsilon$ et $\varepsilon^{\prime}$.

$\left[I(V)\right.$ solar cell characteristic for two sunlight levels $\varepsilon$ and $\varepsilon^{\prime}$.]

$P_{\mathrm{M}}$ en fonction du temps ou par l'utilisation d'un intégrateur, de totaliser l'énergie fournie au cours d'une journée par exemple.

De plus, l'appareil permet de tracer les caractéristiques courant-tension $I(V)$ ou de puissance

$$
P=V I=f(V)
$$

de la cellule à une fréquence de $20 \mathrm{~Hz}$, donc visualisables sur l'écran d'un oscilloscope. Les figures $2 a$ et $2 b$ illustrent le type de tracés obtenus qui comportent les axes de référence $I, V$ et $P, V$ et aussi deux axes $Y, X$ 

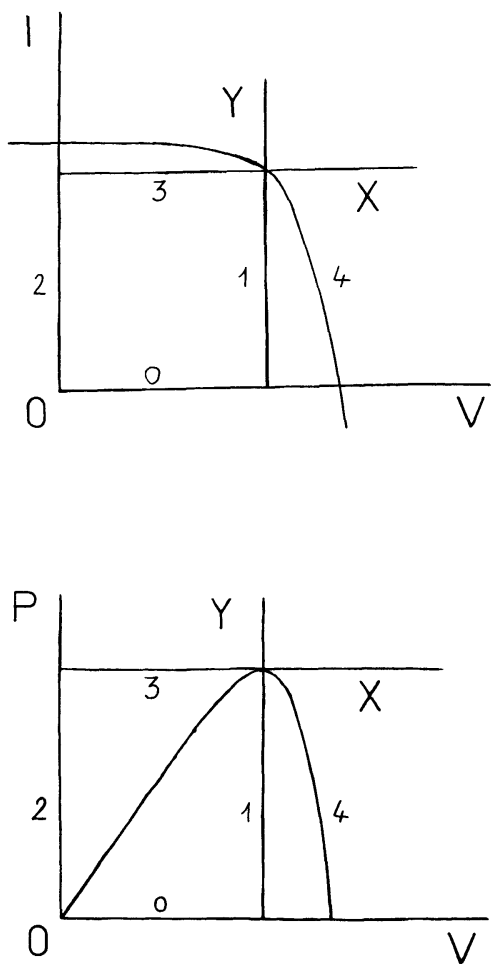

Fig. 2. - Caractéristiques tracées par l'appareil : (a) courant-tension $I(V)$, (b) puissance-tension $P(V)$, les chiffres $(0,1,2,3,4)$ correspondent aux phases successives du tracé.

[Traces given by the apparatus : (a) $I(V)$ characteristic, (b) power versus voltage $P(V)$, numbers $(0,1,2,3,4)$ correspond to different phases of the trace.]

parallèles aux premières dont les positions en abscisse et en coordonnée respectivement sont réglables par potentiomètres; les coordonnées du point d'intersection $\mathbf{M}$ peuvent être lues sur un voltmètre branché aux bornes de sortie prévues à cet effet. Lorsque le point $\mathbf{M}$ coïncide avec un point des caractéristiques $I(V)$ ou $P(V)$, il est possible donc de faire une lecture meilleure qu'à l'oscilloscope, des coordonnées du point.

Finalement, l'appareil présente 3 modes de fonctionnement :

- En mode O, la fréquence est de $20 \mathrm{~Hz}$ pour un tracé complet qui comporte les axes $I, V$ ou $P, V$, les axes réglables $X, Y$ et la caractéristique $I(V)$ ou $P(V)$; l'ensemble est donc visualisable sur l'écran d'un oscilloscope pendant que s'effectue la mesure de $P_{\mathrm{M}}, V_{\mathrm{M}}, I_{\mathrm{M}}$.

- En mode $\mathrm{T}$, un générateur de très basse fréquence $f \simeq 0,1 \mathrm{~Hz}$, permet un tracé précis de la caractéristique $I(V)$ ou $P(V)$ sur une table traçante.

- En mode M, les positions successives du point de fonctionnement sont obtenues manuellement par potentiomètre.

Dans la $2^{\mathrm{e}}$ partie, nous donnerons une vue synoptique des différentes fonctions de l'appareil qui seront étudiées, plus en détail élément par élément dans la $3^{\mathrm{e}}$ partie. Enfin, la $4^{\mathrm{e}}$ partie donnera quelques résultats caractéristiques qui illustreront le fonctionnement de l'appareil.

2. Schéma bloc. - Une vue synoptique est représentée sur la figure 3 . En mode 0, l'oscillogramme total est obtenu par un cycle de cinq phases distribué par un séquenceur constitué par une horloge de fréquence approximative $100 \mathrm{~Hz}$ associé à un diviseur par 5. Ce dernier commande les 2 commutateurs analogiques donnant les tensions de sortie horizontale $X_{0}$ et verticale $Y_{0}$. Chaque phase dure environ $1 / 100$ de seconde, le tracé complet est donc obtenu en $1 / 20$ de seconde.

Sur les bornes des commutateurs numérotés de 0 à 4 , arrivent les cinq phases dont nous donnons ci-dessous les commutations successives :

$\begin{array}{ccll}\frac{\text { Phase }}{0} & \begin{array}{c}\text { Entrée } X \\ \text { rampe }\end{array} & \begin{array}{l}\text { Entrée } Y \\ \text { tension } \\ \text { réglable }\end{array} & \begin{array}{l}\text { mampe } \\ \text { rame horizontal } \\ \text { droite verticale }\end{array} \\ 2 & \begin{array}{c}\text { masse } \\ \text { rampe }\end{array} & \begin{array}{l}\text { rampe } \\ \text { tension } \\ \text { réglable }\end{array} & \begin{array}{l}\text { axe vertical } \\ \text { droite horizontale }\end{array} \\ 3 & V & I \text { ou } P & \text { caractéristique }\end{array}$

Ces phases successives donnent des tracés numérotés de 0 à 4 représentés sur la figure 2 . Pour obtenir les droites et axes horizontaux et verticaux des phases 0,1 , 2,3 , nous avons réalisé un générateur de rampe synchronisé par l'horloge et donnant des tensions en

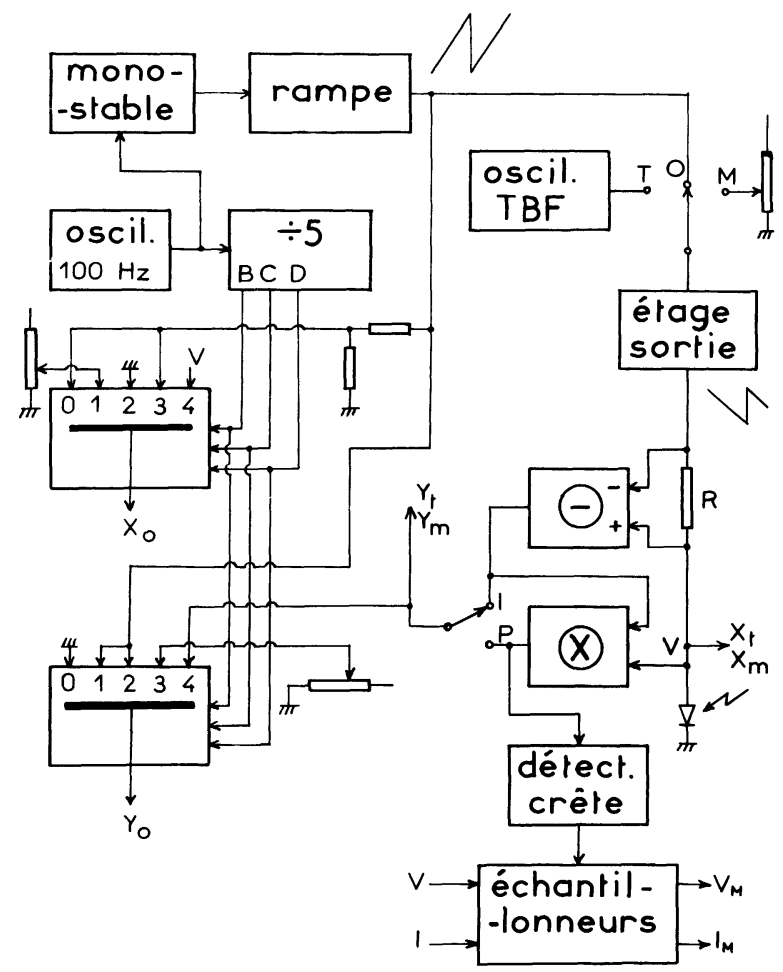

Fig. 3. - Vue synoptique de l'appareil.

[Schematic diagram of the apparatus.] 
dents de scie variant entre 0 et $+6 \mathrm{~V}$. La phase 4 est obtenue par l'intermédiaire d'un amplificateur de puissance sur lequel est appliqué le signal $(0,+6 \mathrm{~V}) \mathrm{du}$ générateur de rampe et qui délivre une tension proportionnelle mais décalée, variant de $+0,8$ à $-2 \mathrm{~V}$ environ. Cette dernière est en série avec une résistance $R$ et la photopile. A ces variations de tension, correspondent des variations de courant dans la photopile ou ce qui revient au même, dans la résistance $R$ qui sont détectées par un circuit de différence.

Les variations de puissance sont obtenues par un circuit multiplieur. La puissance maximum $P_{\mathrm{M}}$ est déterminée par un détecteur de crête, lequel pilote deux échantillonneurs, fournissant les valeurs $V_{M}$ et $I_{\mathrm{M}}$ correspondant à ce maximum.

3. Description de l'appareil. - Dans ce paragraphe, nous allons décrire successivement le fonctionnement électronique des différents blocs du schéma d'ensemble de la figure 3.

3.1 SÉQUENCEUR ET GÉNÉRATEUR DE RAMPE. Le schéma du circuit électronique est représenté sur la figure 4. L'horloge $100 \mathrm{~Hz}$ est constitué par le circuit intégré CI1 qui est un NE 555 alimenté par $+8 \mathrm{~V}$ et $-8 \mathrm{~V}$. La sortie attaque le CI2 qui est un diviseur par 5 , alimenté aussi entre $+8 \mathrm{~V}$ et $-8 \mathrm{~V}$.

Le générateur de rampe est réalisé par le $\mathrm{CI} 3$ (NAND) monté en monostable et excité par CI1 par l'intermédiaire de $R_{3}-\mathrm{D}_{1}$, car CI3 est alimenté entre masse et $+8 \mathrm{~V}$. A chaque flanc descendant du signal d'horloge, donc à chaque changement de phase, le monostable est déclenché; pendant sa période, d'une centaine de $\mu \mathrm{s}$, le condensateur $C 4$ se décharge dans $\mathrm{T}_{2}$. Puis le monostable retourne à son état stable, et $C 4$ se charge linéairement par le générateur de courant $\mathrm{T}_{1}$, d'où l'obtention de la rampe, dont la résistance ajustable $X_{1}$ permet de régler la hauteur. La résistance $R_{9}$ sert à ralentir la vitesse de décharge afin de ne pas imposer des variations trop brusques à l'entrée du multiplieur qui peuvent entraîner des instabilités.

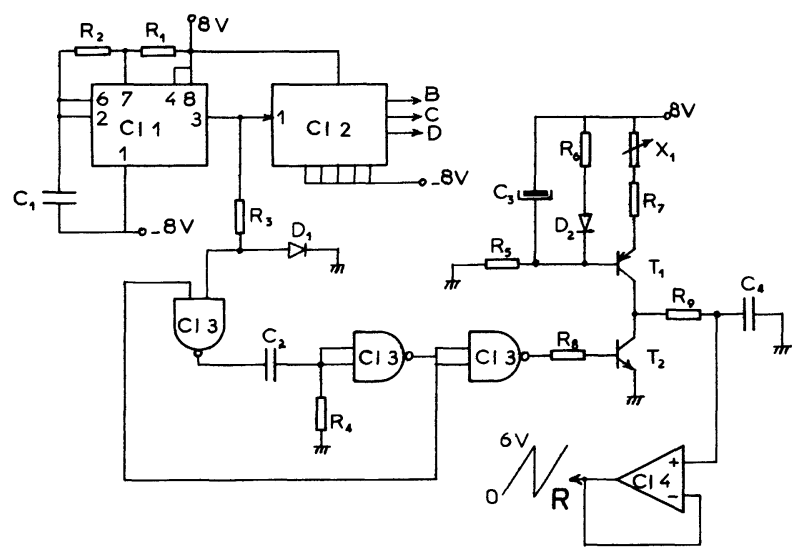

Fig. 4. - Séquenceur et générateur de rampe.

[Relaxation oscillator.]
3.2 L'OSCILlateur très basSe Fréouence. - Ce générateur est représenté sur la figure 5 . Il est constitué de 2 circuits intégrés dont le premier, CI5a est monté en oscillateur à relaxation. Le signal pseudo triangulaire obtenu sur son entrée inverseuse est translatée et légèrement amplifiée par le second $\mathrm{CI} 5 \mathrm{~b}$ pour délivrer un signal évoluant entre 0 et $+6 \mathrm{~V}$ à une fréquence de $0,1 \mathrm{~Hz}$ environ.

3.3 L'ÉTAGe de PUisSANCE. - Cet étage de sortie, dont le schéma est celui de la figure 6 , sert à l'excitation de la photopile à un niveau convenable de courant. Il est constitué du CI6 associé aux transistors $T_{3} a^{a} T_{8}$; l'ensemble est équivalent à un amplificateur opérationnel à fort courant de sortie, de gain voisin de - 1/2. En mode $O$, il est attaqué par la rampe disponible à la sortie de CI4 et donne une tension de sortie évoluant entre $0,8 \mathrm{~V}$ et $-2 \mathrm{~V}$, la diode Zéner $Z_{1}$ apportant un décalage du niveau de la rampe de sortie. A noter la capacité C8 découplant CI6 aux bornes mêmes de ce circuit. Avec ce schéma et les transistors utilisés, il est possible d'atteindre $2 \mathrm{~A}$ en sortie. En mode $T$, l'étage de puissance est attaqué par le générateur TBF de la figure 5. En mode $M$, les rampes sont

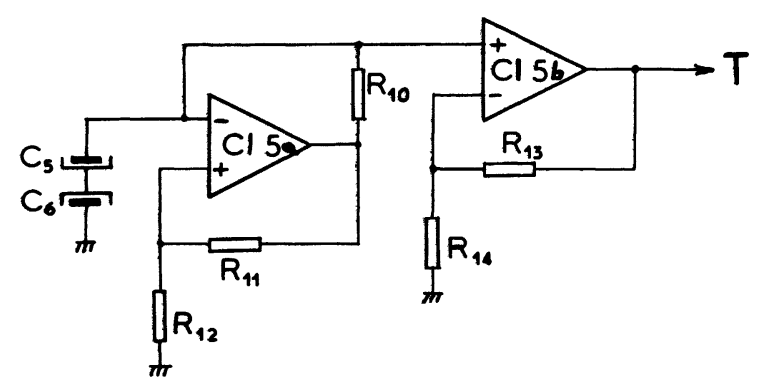

Fig. 5. - Générateur très basse fréquence.

[Ultra low frequency generator.]

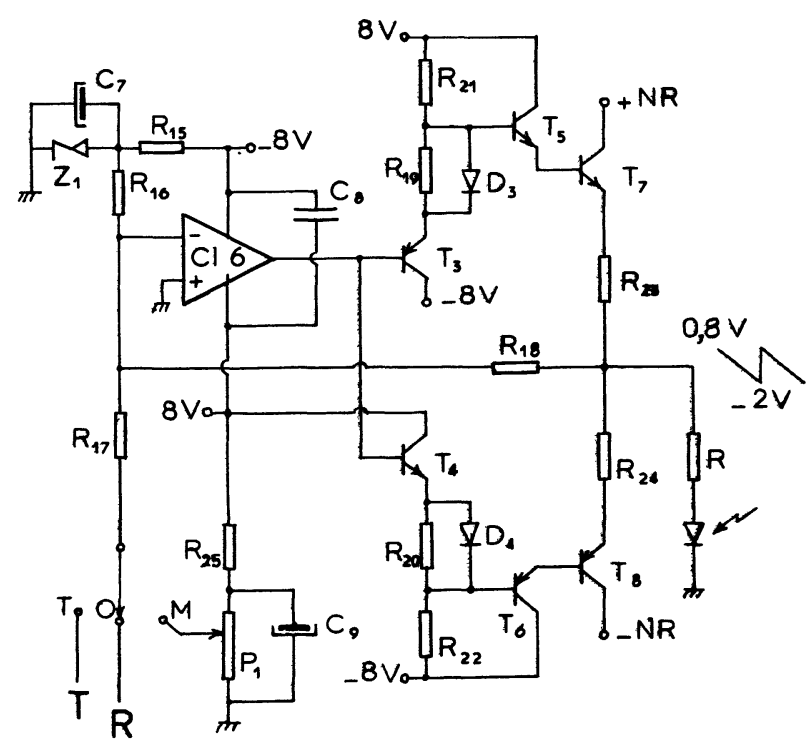

Fig. 6. - Etage de puissance.

[Power stage.] 
remplacées par une tension commandée manuellement par l'intermédiaire du potentiomètre $P_{1}$.

3.4 Différence et multiplication. - Les schémas correspondants à ces étages sont donnés en figure 7 .

Le circuit de différence est réalisé avec les deux amplificateurs opérationnels $\mathrm{CI} 7 \mathrm{a}$ et $\mathrm{CI} 7 \mathrm{~b}$; on obtient en fait en sortie de CI7b, une tension égale à :

$$
V\left(1+\frac{R_{29}}{R_{28}}\right)-V^{\prime}\left(\frac{R_{29}}{R_{28}}+\frac{R_{29}}{R_{28}} \cdot \frac{R_{27}}{R_{26}}\right) .
$$

Si les résistances $R_{26}, R_{27}, R_{28}$ et $R_{29}$ sont rigoureusement égales, on a :

$$
2\left(V-V^{\prime}\right)=2 R I
$$

I étant le courant qui traverse la photopile.

Les résistances sont des éléments à couche métallique de valeur $10 \mathrm{k} \Omega$ à $1 \%$ et triés de telle façon à vérifier la condition :

$$
\frac{R_{29}}{R_{28}}=\frac{R_{26}}{R_{27}} \text { à } 1 \% \text { près . }
$$

Les circuits multiplieurs étant d'un prix relativement élevé, nous avons donc décidé d'en réaliser un à faible coût avec des composants très courants. Nous avons, pour cela, utilisé deux amplificateurs opérationnels doubles $\mathrm{CI} 8$ et $\mathrm{CI} 9$ et un transistor multiple CI10 comprenant les transistors $\mathrm{Ta}, \mathrm{Tb}, \mathrm{Tc}$ et Td. D'après le schéma, on voit que :

$$
\left(V_{\mathrm{be}}\right)_{\mathrm{a}}+\left(V_{\mathrm{be}}\right)_{\mathrm{d}}=\left(V_{\mathrm{be}}\right)_{\mathrm{c}}+\left(V_{\mathrm{be}}\right)_{\mathrm{d}} .
$$

En appliquant la loi d'Ebers et Moll, il vient :

$$
\begin{aligned}
& \frac{k T}{q} \cdot \log \frac{V}{R_{30} I_{\mathrm{s}}}+\frac{k T}{q} \cdot \log \frac{2 R I}{R_{31} I_{\mathrm{s}}}= \\
& \quad=\frac{k T}{q} \log \cdot \frac{V_{\mathrm{Z2}}}{\left(R_{32}+X_{2}\right) I_{\mathrm{s}}}+\frac{k T}{q} \cdot \log \frac{V_{\mathrm{s}}}{R_{33} I_{\mathrm{s}}}
\end{aligned}
$$

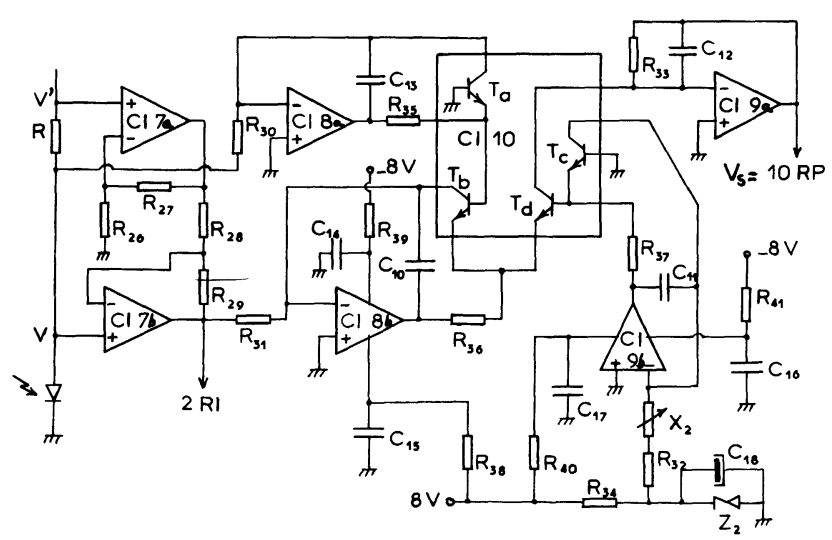

Fig. 7. - Circuits de différence et de multiplication.

[Difference circuit and multiplication circuit.] avec :

$I_{\mathrm{s}} \quad$ : courant de saturation

$V_{\mathrm{Z}_{2}}$ : tension aux bornes de la diode Zéner $\mathrm{Z}_{2}$

$V_{\mathrm{s}}:$ tension de sortie.

D'où :

$$
\frac{2 R I \cdot V}{R_{31} R_{30}}=\frac{V_{\mathrm{Z} 2} \cdot V_{\mathrm{s}}}{R_{33}\left(R_{32}+X_{2}\right)} .
$$

Compte tenu que $R_{31}=R_{30}=R_{33}$, on trouve :

$$
V_{\mathrm{s}}=2 R P \cdot \frac{R_{32}+X_{2}}{V_{\mathrm{Z} 2} \cdot R_{30}} .
$$

Nous avons ajusté $X_{2}$ pour que :

$$
V_{\mathrm{s}}(\mathrm{V})=10 R_{(\Omega)} \cdot P_{(\mathrm{W})} .
$$

Ce multiplieur ne peut opérer que dans un quadrant ce qui est suffisant dans cette application. Un câblage soigné et des précautions particulières doivent être prises pour éviter les oscillations spontanées : contreréactions sur les amplificateurs opérationnels avec des condensateurs $C_{10}, C_{11}, C_{12}, C_{13}$, évitant la réaction par les transistors grâce à $R_{35}, R_{36}, R_{37}$. De plus, les circuits intégrés $\mathrm{CI} 8$ et $\mathrm{CI} 9$ sont alimentés sur leurs bornes positive et négative par des cellules R-C.

3.5 LeS COMmutateurs ÉlectroniQues. - Les sorties $X_{0}$ et $Y_{0}$ émergent de commutateurs électroniques constitués par les circuits intégrés CI11 et CI12 de la figure 8 . Ils sont à 8 positions, dont les entrées 0 à 4 , recevant les signaux de travail déjà décrits au paragraphe 2 , sont successivement commutés par les entrées de sélection reliées aux sorties BCD de CI2. Le commutateur CI11, relatif à $X_{0}$, ne reçoit qu'une fraction de la rampe, puisque la tension aux bornes d'une photopile ne dépasse guère $0,7 \mathrm{~V}$. CI12 reçoit $I$ ou $P$ au choix d'un inverseur. Les potentiomètres $P_{2}$ et $P_{3}$ permettent de régler les positions des droites de repérage, la course de $P_{3}$ étant naturellement plus réduite.

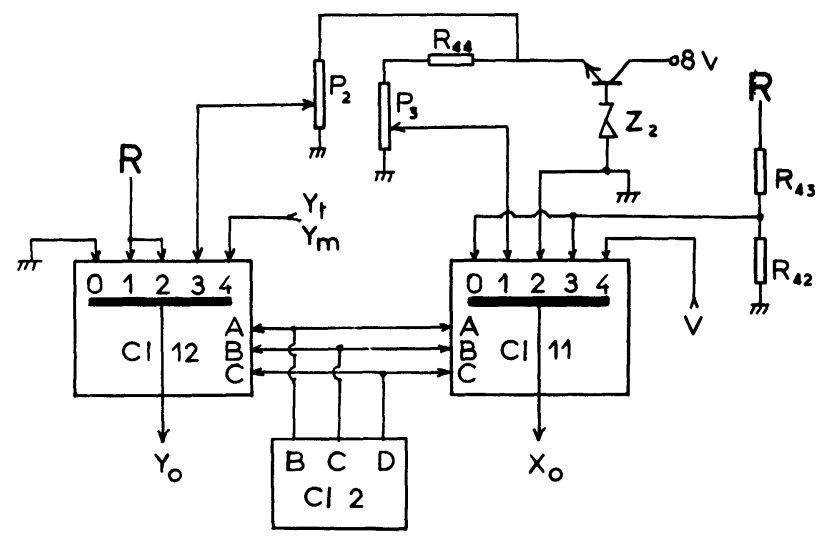

Fig. 8. - Commutateurs électroniques.

[Electronic switch.] 
3.6 COORDONNÉES DU POINT $\boldsymbol{P}_{\mathrm{M}}$. - L'appareil doit fonctionner en mode $O$. La figure 9 donne le schéma du circuit qui permet d'obtenir la puissance $P_{M}$ ainsi que ses coordonnées $V_{\mathrm{M}}$ et $I_{\mathrm{M}}$. $P_{\mathrm{M}}$ est obtenue, à partir du signal de puissance par un détecteur de crête sans seuil formé de CI13, $D_{5}, C_{19}$ et $R_{45}$. La. constante de temps $C_{19} R_{45}$ de l'ordre de 1 seconde, est choisie pour que la sortie $P_{\mathrm{M}}$ puisse suivre presque sans inertie les variations d'ensoleillement, en restant assez grande pour que la décharge de $C_{19}$ dans $R_{45}$ reste très faible entre deux réajustements successifs distants de 1/100 de seconde. CI14 sort $P_{\mathrm{M}}$ à basse impédance; il est pourvu d'une correction d'offset à régler sous faible luminosité et son gain très légèrement supérieur à l'unité est à régler par $X_{3}$ en forte luminosité pour rattraper la décharge de $C_{19}$ dans $R_{45}$. De plus, lors de chaque passage au maximum, l'impulsion positive obtenue en sortie de CI13 est mise en forme par $T_{10}$ et $T_{11}$. Depuis $T_{11}$, cette impulsion commande les interrupteurs de CI15, d'où l'échantillonnage de $V$ et $I$ à chaque passage par $P_{\mathrm{M}}$.

4. Résultats. - Les photos d'oscillogrammes données sur les figures $10 a$ et $10 b$ représentent respecti-

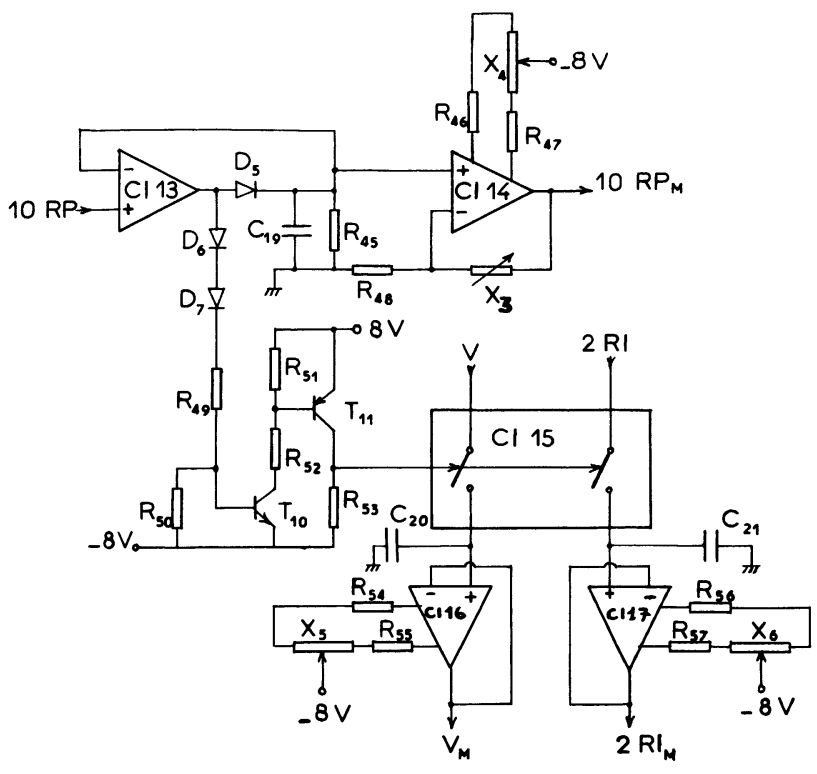

Fig. 9. - Circuits donnant les coordonnées du point de puissance maximum $\boldsymbol{P}_{\mathbf{M}}$.

[Electronic circuit giving the values $I_{\mathrm{M}}, V_{\mathrm{M}}, P_{\mathrm{M}}$ of the maximum power point.]
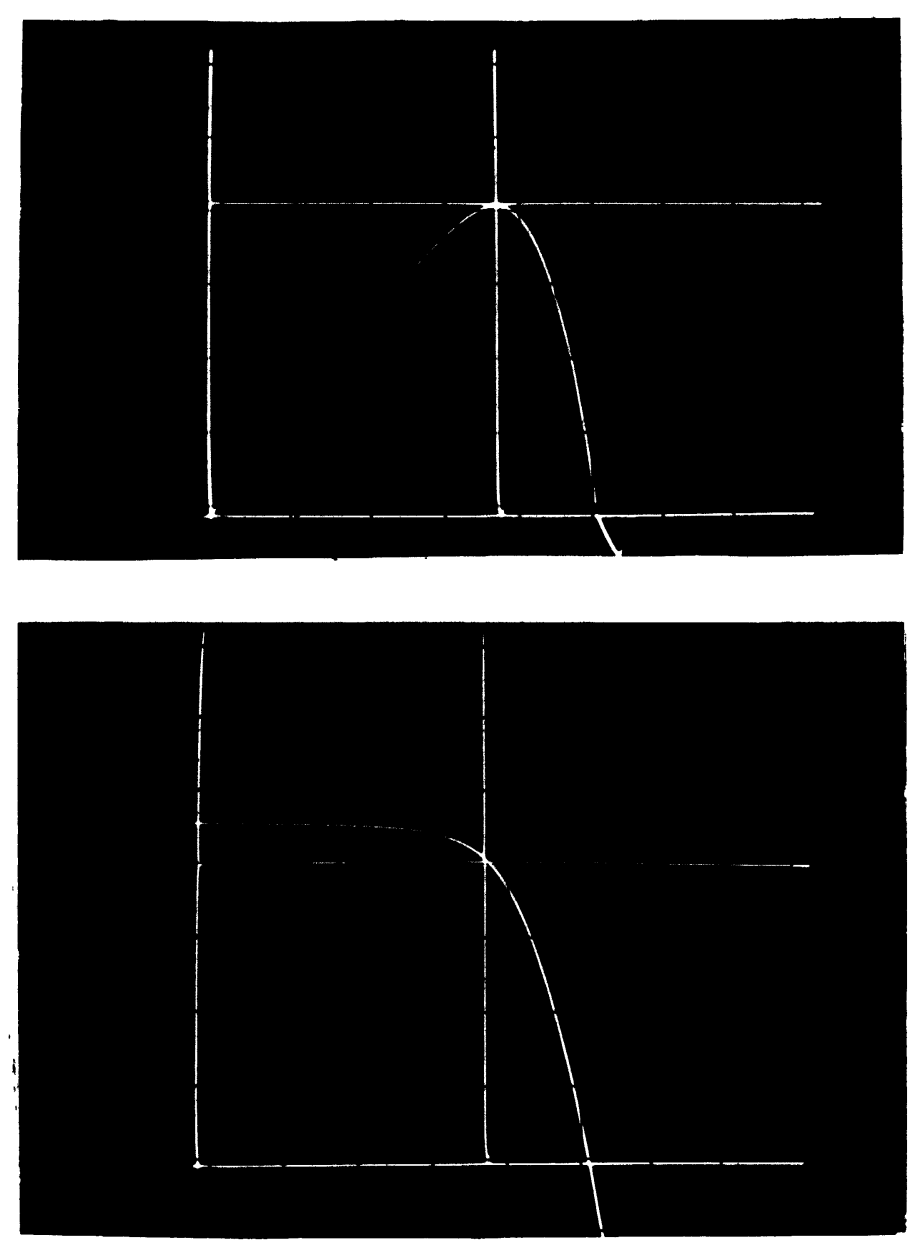

Fig. 10. - Oscillogrammes : a) de la courbe de puissance $P(V)$, b) de la caractéristique $I(V)$.

[Oscillograms : a) $P(V)$ power curve, b) $I(V)$ characteristic.] 
vement la courbe de puissance $P(V)$ et la caractéristique courant-tension $I(V)$ d'une photopile solaire au silicium. Les axes $X$ et $Y$ ont été réglées sur la courbe de puissance de la figure $10 a$ de telle façon que leur point d'intersection coïncide avec le maximum de la courbe, soit $P=P_{M}$. Pour cette cellule et pour l'éclairement défini au moment de la photographie, nous avons mesuré en abscisse une tension $V_{M}$ égale à $0,41 \mathrm{~V}$ et en ordonnée, une tension $V_{\mathrm{s}}$ de $4,51 \mathrm{~V}$; ayant utilisé une résistance $R=1,1 \Omega$ la puissance maximale s'obtient par la formule :

$$
\begin{aligned}
& P_{\mathrm{M}}(\mathrm{W})=V_{\mathrm{s}}(\mathrm{V}) / 10 R(\Omega), \\
& \text { soit finalement } \quad P_{\mathrm{M}} \simeq 410 \mathrm{~mW} .
\end{aligned}
$$

La photographie de la figure $10 b$ a été prise immédiatement après celle de la figure $10 a$; le courant maximum $I_{M}$ se détermine en gardant l'axe $Y$ à sa position précédente et en amenant l'axe $X$ au point d'intersection de l'axe $Y$ avec la caractéristique $I(V)$. Nous avons noté à la sortie du circuit de différence une tension égale à $2,15 \mathrm{~V}$ soit un courant $I_{\mathrm{M}}=\frac{2,15}{2 R} \simeq 0,97 \mathrm{~A}$ d'où une puissance maximale en ce point égale à $P_{\mathrm{M}}=V_{\mathrm{M}} \cdot I_{\mathrm{M}}=0,41 \times 0,77$, soit $P_{\mathrm{M}}=400 \mathrm{~mW}$, valeur très voisine de celle déterminée avec le circuit multiplieur précédent. Il est bien évident que les coordonnées de n'importe quel autre point des courbes $I(V)$ et $P(V)$ peuvent être obtenues par déplacement des axes $X$ et $Y$ réglables par potentiomètres, et de telle manière que le point d'intersection des axes $X$ et $Y$ coïncide avec le point choisi de la courbe $P(V)$ ou $I(V)$. Rappelons que lors de telles mesures, l'appareil continue de fournir à tout instant sur des sorties indépendantes, les valeurs de $P_{\mathrm{M}}, V_{\mathrm{M}}, I_{\mathrm{M}}$.

Enfin, l'instrument étant conçu avec une position « tracé " de courbe à très basse fréquence, nous avons reproduit en figure 11, un relevé sur table traçante de la courbe de puissance $P(V)$ et de la caractéristique $I(V)$. On note l'intérêt d'un tel diagramme qui permet de déterminer rapidement l'abscisse $V_{M}$ correspondant au point $P=P_{M}$ sur la courbe $P(V)$ et donc l'ordonnée $I_{\mathrm{M}}$ sur la caractéristique $I(V)$ soit les coordonnées $I_{\mathrm{M}}$, $V_{M}$ du point de puissance maximum.

Ici, nous avons utilisé une résistance $R=\frac{2,2}{3} \Omega$;

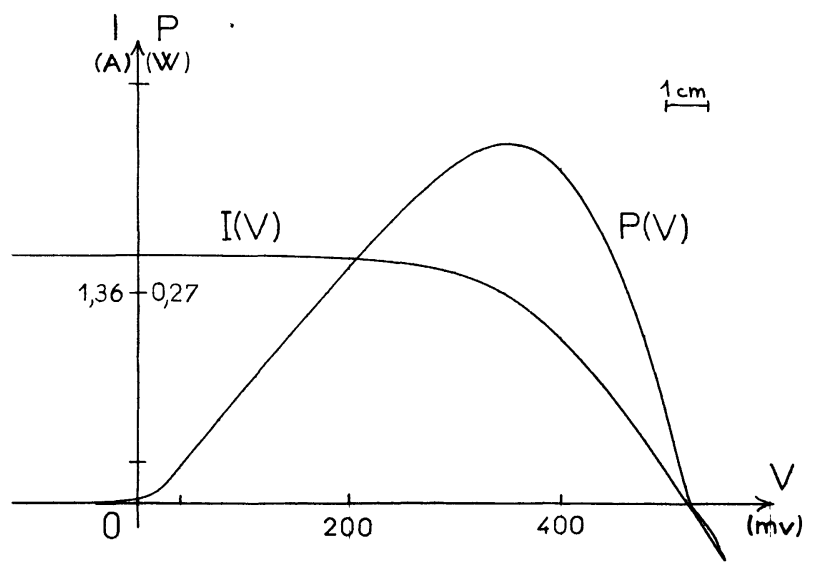

Fig. 11. - Tracés sur table $X-Y$ des caractéristiques courant-tension $I(V)$ et puissance $P(V)$.

$[X-Y$ traces of $I(V)$ characteristic and power $P(V)$ curve.]

les valeurs des tensions sur les axes $X$ et $Y$ étaient respectivement $40 \mathrm{mV} / \mathrm{cm}$ et $400 \mathrm{mV} / \mathrm{cm}$ d'où les valeurs correspondantes de $0,272 \mathrm{~A} / \mathrm{cm}$ pour l'axe des courants $I$ et $54 \mathrm{~mW} / \mathrm{cm}$ pour l'axe des puissances $P$.

5. Conclusion. - La charge active pour photopiles solaires que nous avons réalisée, présente un certain nombre de particularités que nous rappelons brièvement :

- Tracé manuel, à très basse fréquence ou oscilloscopique des courbes de courant-tension $I(V)$ ou de puissance $P(V)$.

- Détermination par repérage d'axes, des coordonnées des points de chaque courbe et en particulier du point de puissance maximum.

- En mode oscilloscopique, détermination permanente de la puissance maximum $P_{M}$ et de ses coordonnées tension $V_{\mathrm{M}}$ et courant $I_{\mathrm{M}}$.

De plus, nous avons conçu cet appareil avec le souci permanent d'un faible prix de revient. Nous pensons avoir atteint notre objectif en ayant imaginé un circuit multiplieur utilisant des composants classiques de faible coût donnant toute satisfaction. 